\title{
ASCO GU 2018: Neue Behandlungsoptionen für Prostatakarzinompatienten
}

Auf dem diesjährigen ASCO GU-Kongress in San Francisco spielten insbesondere die Prostatakarzinome eine prominente Rolle. Die Ergebnisse zweier Studien zu nicht-metastasiertem kastrationsresistentem Prostatakarzinom (nmCRPC) wurden mit Spannung erwartet und erfüllten die Erwartungen der anwesenden Mediziner. Eine neue Auswertung von Daten der STAMPEDEStudie gibt Anlass zu weiteren Diskussionen über Therapieoptionen bei Patienten mit fortgeschrittenem hormonsensitivem Prostatakarzinom.

Bei dem jährlich stattfindenden Kongress treffen sich Uroonkologen aus der ganzen Welt, um sich über die neuen Entwicklungen bei der Erforschung von Krebserkrankungen im Urogenitalbereich $\mathrm{zu}$ informieren und über Therapiemöglichkeiten zu diskutieren. Dieses Jahr spielten die Prostatakarzinome eine wichtige Rolle, da es hier aktuelle Daten gab, die mit großer Wahrscheinlichkeit die Therapiepalette erweitern werden.

\section{Zwei Wirkstoffe für nmCRPC Patienten}

Für Patienten, die ein nmCRPC und ein hohes Risiko für die Entwicklung von Metastasen haben, gibt es bislang keine zugelassene Therapieoption. In großen randomisierten Phase-III-Studien wurden 2 Wirkstoffe, Apalutamid in der Studie SPARTAN und Enzalutamid in der Studie PROSPER, in dieser Indikation getestet $[1,2,3]$. Die Patienten gehören zu einer Hochrisikogruppe mit sehr niedrigen PSA (Prostata-spezifisches Antigen)-Verdopplungszeiten und damit einhergehend einem hohen Risiko der Ausbildung von Metastasen. Dieses Risiko korreliert mit erhöhter Morbidität und Mortalität und ist daher ein bisher nicht abgedeckter medizinischer Bedarf [4].

Beide Studien sind sehr ähnlich angelegt und kommen zu weitestgehend kongruenten Ergebnissen. Bei einer Patientenzahl von über 1000 war das wichtigste Einschlusskriterium eine PSA-Verdopplungszeit von unter 10 Monaten, wobei der Median in beiden Studien unter 6 Monaten lag. Die Patienten wurden 2:1 (Verum: Placebo) randomisiert und der primäre Endpunkt war das metastasenfreie Überleben (MFS), gemessen mittels radiographischer Verfahren. Sowohl Apalutamid als auch Enzalutamid zeigten hier signifikant überlegene Werte mit Hazard Ratios von 0,28 bezie- hungsweise 0,29 ( $\mathrm{p}<0,0001)$. Dabei verlängerte sich das metastasenfreie Überleben in beiden Studien um ungefähr 2 Jahre $(24,3$ respektive 21,9 Monate).

In den sekundären Endpunkten setzten die Studien leicht unterschiedliche Akzente. Die SPARTAN-Studie (Apalutamid) untersuchte neben dem progressionsfreien Überleben (PFS), dem Gesamtüberleben (OS) und der Zeit bis zum Einsatz einer Folgebehandlung nach Progress, zusätzlich noch die Zeit bis zum symptomatischen Progress (sympProg) und den exploratorischen Endpunkt des zweiten progressionsfreien Überlebens (PFS2), gemessen von Randomisierung bis zur Progression nach Zweitbehandlung. Beide Studien nahmen auch Daten zum PSA-Progress und zur Lebensqualität auf.

Ein Vergleich der bisher gezeigten Studiendaten ist in Tabelle 1 zu sehen. In beiden Studien hielten sich die Nebenwirkungen in zu erwartenden Grenzen. Mit Enzalutamid traten 31\% unerwünschte Ereignisse von Grad 3 und höher auf, gegenüber 23\% im PlaceboArm. Für Apalutamid lagen die Zahlen bei 45\%, respektive 34\%. Es muss bei der Bewertung der Nebenwirkungen berücksichtigt werden, dass das Alter der Studienteilnehmer sehr hoch war mit jeweils bis zu 95 Jahre alten Patienten.

Beide Wirkstoffe gehören zu einer Wirkstoffklasse von modernen Androgenrezeptor-Inhibitoren. Während Enzalutamid bereits für die Erst- und Zweitlinientherapie bei metastasiertem kastrationsresistentem Prostatakarzinom zugelassen ist [5], drängt Apalutamid nun auf eine Erstzulassung. Die Studien geben Anlass zu Hoffnung auf eine baldige Zulassung einer Behandlung bei der nmCRPC Hochrisiko-Patientengruppe.

\section{Docetaxel/ADT-Kombination bei fortgeschrittenem Prostatakarzinom}

Zur STAMPEDE-Studie, auf deren Basis Patienten mit metastasiertem hormonsensitivem Prostatakarzinom seit einiger Zeit die Kombination aus Docetaxel plus dem bis dahin üblichen Standard Androgendeprivationstherapie (ADT) erhalten, wurden prognostische Kalkulationen zu den qualitätskorrigierten Lebensjahren (QALY) und der Kosteneffektivität bezogen auf die Lebensqualität (ICER) vorgestellt [6, 7]. Bei beiden Endpunkten konnten sowohl

\section{KARGER}

(c) 2018 S. Karger GmbH, Freiburg 
Table 1. Gegenüberstellung der Studienergebnisse der PROSPER- und SPARTAN-Studie $[1,2,3]$

\begin{tabular}{|c|c|c|c|c|c|c|}
\hline & \multicolumn{3}{|l|}{ PROSPER } & \multicolumn{3}{|l|}{ SPARTAN } \\
\hline & $\begin{array}{l}\text { Enzalutamid + } \\
\text { ADT }\end{array}$ & $\begin{array}{l}\text { Placebo + } \\
\text { ADT }\end{array}$ & Hazard Ratio & $\begin{array}{l}\text { Apalutamid + } \\
\text { ADT }\end{array}$ & $\begin{array}{l}\text { Placebo + } \\
\text { ADT }\end{array}$ & Hazard Ratio \\
\hline MFS & 36,6 & 14,7 & $0,29(0,24-0,35)$ & 40,5 & 16,6 & $0,27(0,22-0,34)$ \\
\hline Zeit bis zur Folgetherapie & 39,6 & 17,7 & $0,21(0,17-0,26)$ & $N R$ & $N R$ & $0,44(0,29-0,66)$ \\
\hline PSA-Progress & 37,2 & 3,9 & $0,07(0,05-0,08)$ & $N R$ & 3,7 & $0,06(0,05-0,08)$ \\
\hline OS & $N R$ & $N R$ & $0,8(0,58-1,09)$ & $N R$ & 39 & $0,7(0,47-1,04)$ \\
\hline sympProg & ND & ND & ND & NR & NR & $0,45(0,32-0,63)$ \\
\hline PFS & ND & ND & ND & 40,5 & 14,7 & $0,29(0,24-0.36)$ \\
\hline
\end{tabular}

In kursiv: Endpunkt noch nicht erreicht, MFS: metastasenfreies Überleben, OS: Gesamtüberleben, sympProg: symptomatische Progession, PFS: progessionsfreies Überleben, NR: not reached, ND: not determined, alle Angaben in Monaten.

für die Patienten mit (M1) als auch für die Patienten ohne (M0) Metastasen Vorteile der Kombinationsbehandlung im Vergleich zur ADT gezeigt werden. Die QALY erhöhten sich um 0,51 (M1) beziehungsweise 0,39 Jahre (M0) und auch die Kosteneffektivität fiel für beide Patientengruppen positiv für die Kombinationsthera- pie aus. Die Autoren des Abstracts empfehlen daher diese Form der Behandlung auch für das nicht-metastasierte fortgeschrittene hormonsensitive Prostatakarzinom.

Dr. med. Gerhard Emrich

\section{Literatur}

1 Smith MR, et al. N Engl J Med 2018:doi:10.1056/ NEJMoa1715546.

3 Hussain M, et al. ASCO GU 2018:abstr 3.

4 Smith MR, et al. J Clin. Oncol. 2013;31:3800-6.

5 Beer TM, et al. N Engl J Med. 2014;371(5):424-433.
6 James ND, et al. The Lancet. 2016;387(10024): 1163 1177

7 James ND, et al. ASCO GU 2018:abstr 162. 